\title{
Theoretical Study of Spin Conduction in the Ni/DTE/Ni nanohybrid
}

\author{
Kanchan Ulman ${ }^{1, a}$, Mighfar Imam ${ }^{1, b}$, Shobhana Narasimhan ${ }^{1, c}$, Anders Odell ${ }^{2, d}$ \\ and Anna Delin ${ }^{2,3,{ }^{*} \mathrm{e}}$
}

${ }^{1}$ Theoretical Sciences Unit, Jawaharlal Nehru Centre for Advanced Scientific Research, Jakkur,

Bangalore, 560064, India

${ }^{2}$ Royal Institute of Technology (KTH), School of Information and Communication Technology, Electrum 229, SE-16440 Kista, Sweden
${ }^{3}$ Department of Physics and Astronomy, Uppsala University, Box 516, SE-75120 Uppsala, Sweden
aulman@jncasr.ac.in, ํmighfar@jncasr.ac.in, cshobhana@jncasr.ac.ine, dodell@kth.se,
*eannadel@kth.se ( ${ }^{*}$ Corresponding Author)

Keywords: Spin molecular electronics; spin conduction; dithienylethene: density functional calculations; non-equilibrium Green function calculations

\begin{abstract}
The photoswitching molecule dithienylethene (DTE) is an interesting candidate for constructing optoelectronic molecular devices since it can be made to switch between a closed and an open conformation using light. We here report computations, based on density functional theory (DFT) and the non-equilibrium Green function (NEGF) method, of the spin-resolved conductance of the two DTE isomers attached to spin-polarized nickel leads. Results are compared and contrasted to those of other contact materials (nonmagnetic $\mathrm{Ni}, \mathrm{Ag}$, and $\mathrm{Au}$ ), analyzing the physical origins of the various features in the transmission function. It was found rather surprisingly, that the two spin channels in the Ni/DTE/Ni device have almost identical $I$ - $V$ characteristics, despite one channel being $d$-dominated and the other one $s$-dominated. It was also observed that the Ni-based device exhibits a sustained high conductance ratio also for high bias - a property that may be of relevance in future device design. Furthermore, two computational schemes for calculating the conductance were compared and analyzed. It was found that even for very small bias the molecular orbital polarization was decisive for spin-related properties such as the spin current ratio and magneto-resistance in the Ni/DTE/Ni device.
\end{abstract}




\section{Introduction}

Molecular electronics is an exciting, promising and fast-growing field because it offers the possibility of miniaturizing electronic devices at low cost, sidestepping the barriers imposed by conventional semiconductor technology and the top-down approach to building devices [1]. Among the many classes of molecules that are of interest for molecular electronics applications, an intriguing class is that of reversible photoswitching molecules, which can be switched between two stable conformations by light. In this paper, the transport characteristics of one such molecule, dithienylethene (DTE) is studied in the context of spin-polarized leads. The DTE molecule lends itself very well as model system in systematic investigations of molecular transport properties since one confirmation has metallic-like conductance, whereas the other one has a much lower, tunnellinglike conductance. This enables comparisons of the two transport regimes in a well-controlled setting involving minimal chemical manipulation of the system. Ni as lead material was chosen because of its high degree of spin-polarization at the Fermi level. Ni is a so-called strong ferromagnet [2] with virtually only one spin channel in the $d$-band represented at the Fermi level. The structure of the DTE molecule (see Fig. 1) consists of three carbon rings: a central six-fold ring, connected on either side to two pentagonal rings (four carbon atoms and a sulfur atom), with sulfur atoms on either side that may bind to metal surfaces (leads), allowing an electric voltage to be applied over the molecule. Under the action of visible light, one of the carbon-carbon bonds in the central ring is broken, switching the molecule from a highly conducting to a low-conductance conformation. 


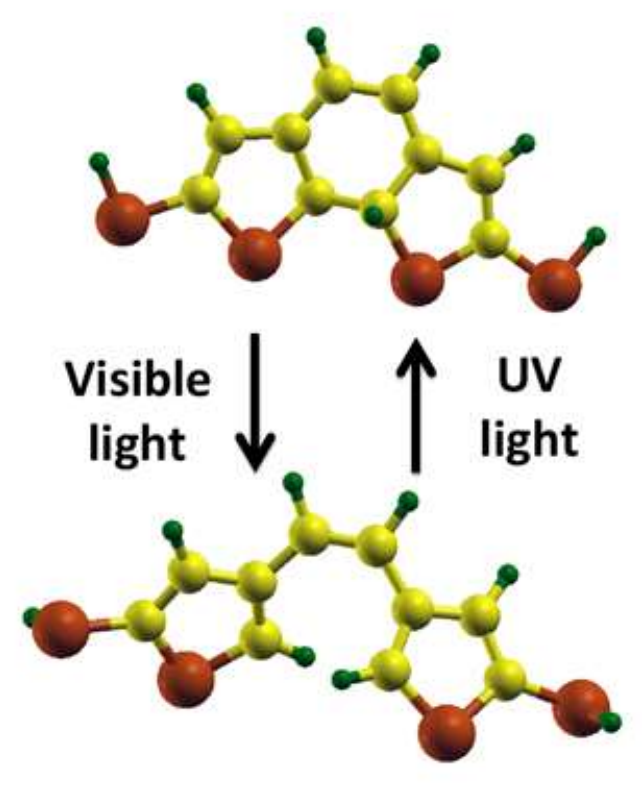

Fig. 1. (Color online) The two geometrical conformations of the DTE molecule, closed conformation at the top and the open conformation below. Color code: yellow balls $\mathbf{C}$, large red balls $\mathbf{S}$ and green balls $\mathbf{H}$. Image created with XCrySDen [3].

In this work, the focus lies in particular on how spin-polarization of the lead material, and also the electronic structure of the lead material in general, may affect the transport properties of this molecule. Specifically, the case of spin-polarized Ni leads is compared to three different nonmagnetic lead materials - $\mathrm{Au}, \mathrm{Ag}$ and nonmagnetic Ni. In spin-polarized $\mathrm{Ni}$, the majority spin channel has mostly $s$-states at the Fermi level, whereas the minority spin channel is strongly $d$ dominated. Thus, comparing these systems should bring insight into how spin-resolved $s$ - and $d$ states, respectively, may affect the conduction properties of the electrode/DTE/electrode system, with the spin-polarized $\mathrm{Ni}$ case taking a conceptual intermediate position between $\mathrm{Au}$ and $\mathrm{Ag}$ on one hand and nonmagnetic $\mathrm{Ni}$ on the other. These features are brought out by examining the bulk densities of states (DOS) for the four relevant contact materials, shown in Fig. 2. In principle, the sharp features in the DOS mainly arise from $d$-states whereas the broader features arise mainly from $s$-states. In this case this figure shows the total DOS only. The $s$ - and $d$-character of the spin channels at the Fermi level can be confirmed by examining the orbital-resolved DOS (not shown). 
In recent years, there have been several experimental and theoretical studies on the transport characteristics of DTE and closely related molecules. In one set of experiments, where DTE molecules were attached to gold contacts, it was reported that the closing reaction was quenched by the presence of gold [4]. However, several other similar experiments on related molecules for reversible switching between open and closed conformations had been reposted [5-9]. The ratio between the conductance of the closed and open forms of the molecule, when attached to gold contacts was measured and reported [10], a lower limit of 30 of the conductance ratio was determined using pyridine groups linking to the gold leads combined with the technique of repeatedly formed break. There have also been related theoretical calculations of transport properties [9,11-13]; however these were not performed in a self-consistent way or/and did not consider DTE. Earlier work, experimental as well as theoretical, have been performed for the most part with Au as lead material, with the molecule attached with thiol-bonds, i.e., a sulfur bridge. Molecules can be attached also to transition metals using the same type of bond [15]. In earlier work by us [16,17], the conduction and properties of the DTE molecule when attached to Ag, Au and nonmagnetic Ni leads are discussed in more detail. Purely for clarity of the present discussion some of the results in those papers are also illustrated here. 

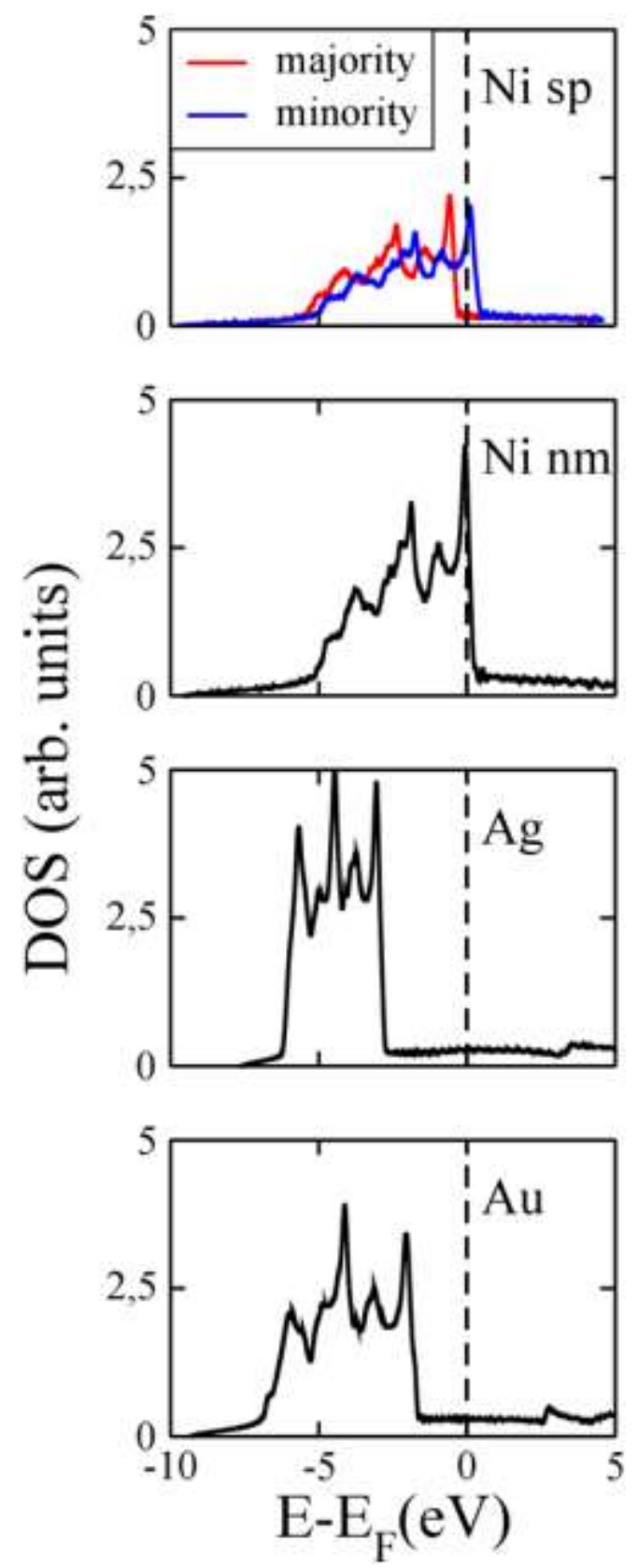

Fig. 2. (color online) Bulk density of states (DOS) for the lead materials discussed in this study. From top to bottom: spin-polarized $\mathrm{Ni}$, nonmagnetic $\mathrm{Ni}, \mathrm{Ag}$ and $\mathrm{Au}$. The red and blue curves denote the majority and minority spin channels, respectively.

\section{Method}

Geometry optimization and electronic structure calculations were performed within the framework of ab initio density functional theory (DFT), making use of the SIESTA code [18], while the transport calculations were performed with the SMEAGOL code [19-21], which interfaces the nonequilibrium Green function (NEGF) scheme with DFT calculations as performed in SIESTA. In the strong-coupling limit, DFT appears to be reliable for transport calculations although perfect 
quantitative agreement with experiment can of course not be expected due to, e.g., limitations in the description of correlation [22]. As a means to analyze trends as well as explaining the origins of observed physical phenomena in the context of molecular conduction, standard DFT-based computations combined with NEGF have proven very useful.

The SIESTA calculations were performed using norm-conserving, nonlocal Troullier-Martins pseudopotentials [23], and a numerical basis set. A double $\zeta$ plus polarization orbital basis set was used for all the atoms in the DTE molecule, while the atoms in the metal leads were treated at the level of single $\zeta$ orbitals for the $d$ electrons and double $\zeta$ orbitals for the $s$ electrons plus a $p$ polarization orbital. Exchange and correlation interactions were treated by the Perdew-BurkeErnzerhof form of the generalized gradient approximation [24].

For the transport calculations, the system was divided into three regions: a left metallic electrode or lead, a scattering region (SR) and a right metallic lead. The two leads were treated as reservoirs at different chemical potentials. (The SR is the region where the scattering processes as well as the electrostatic potential drop take place; it typically consists of the molecule of interest as well as a few layers of atoms from the left and right leads.) Using the SMEAGOL code, the SR Green function and charge density were then calculated in a self-consistent procedure. Once this procedure was converged, the transmission function was evaluated from the Landauer-Büttiker formula [25]. The transmission function depends on the applied bias and the $I-V$ characteristics were thus calculated self-consistently taking into consideration this bias dependence. However, in this work also non-selfconsistent (NSC) calculations of the conductance are presented in order to analyze what errors are introduced if one does not take into account the effect of finite bias on the transmission function. This simplified scheme has been rather popular in the past and appears to give good results in several cases $[9,11]$. Therefore, it is interesting to elucidate under what circumstances the non-self-consistent approach may work and what the reasons for this might be. 
In our calculations, we first obtained the relaxed geometry of the DTE molecule in vacuum, in both the open and closed conformations. The relaxed structure was then placed between the metal leads. The parts of the leads contained within the scattering region were modeled as five layers each of metal stacked in a face-centered-cubic (100) orientation. The metal's lattice constant was chosen to be equal to the experimental value. The sulfur atoms were assumed to bind to the hollow site on the metal surface, which is known to be the stable adsorption geometry. In the results presented in this paper, the distance between the metal and the sulfur atom was kept fixed at $1.9 \AA$ for all cases. While this is indeed the stable adsorption geometry for the $\mathrm{Au}$ and $\mathrm{Ag}$ leads, for $\mathrm{Ni}$ it is an overestimation of the bonding distance, which is actually shorter. For comparison, the bond length was held constant for all the cases considered. We have, however, verified that for the case of $\mathrm{Ni}$, relaxing the adsorption geometry does not alter the results in any qualitative way [17]. Moreover, the work presented here is restricted to the case where both the leads are polarized parallel to one another. For further details of the computational setup, the reader is referred to our previous publications on the subject $[16,17]$.

\section{Results and Discussion}

The main entity to be calculated was how the current through the molecule depends on the applied bias. This gives the so-called $I-V$ characteristics of the system in question. Fig. 3 shows the $I-V$ characteristics for the DTE molecule attached to four lead materials (spin-polarized Ni, nonmagnetic $\mathrm{Ni}, \mathrm{Ag}$, and $\mathrm{Au}$ ), in both the open and closed configurations. The $I$ - $V$ characteristics have been calculated both self-consistently (i.e., the electronic structure of the system is allowed to adapt to the applied bias) and non-self-consistently (i.e., the zero bias transmission function is used in the Landauer-Büttiker formula for all bias voltages). The self-consistent results (left column in Fig. 3) will be addressed first, with a focus on how the conductance was altered as a function of changing the lead material and in particular what happens as the Ni leads were allowed to spin-polarize. It was found that for all four lead materials, the current increases with bias, as expected, although for the 
Ni-based devices, the conductance of the open isomer was almost constant and very low in a large interval, compared to the situation with Au and Ag leads. The DTE in the open configuration also consistently had lower conductance than in the closed configuration. At low bias, the ratio of conductance between the closed and open isomers was around 20 with Au electrodes, 35 for Ag, 40 for nonmagnetic $\mathrm{Ni}$, and over 60 for spin-polarized $\mathrm{Ni}$ electrodes. At $1 \mathrm{~V}$ bias, the corresponding numbers were around 5 for $\mathrm{Au}$ and $\mathrm{Ag}, 50$ for nonmagnetic Ni, and 50 for spin-polarized Ni. Thus, the $\mathrm{Ni} / \mathrm{DTE} / \mathrm{Ni}$ device appears to behave very differently compared to the $\mathrm{Au} / \mathrm{DTE} / \mathrm{Au}$ and $\mathrm{Ag} / \mathrm{DTE} / \mathrm{Ag}$ devices as a function of bias and the Ni-based devices keept their high conductance ratio whereas the $\mathrm{Au}$ and Ag-based ones exhibit a ratio which quickly decreases with bias. Interestingly, in the case of spin-polarized $\mathrm{Ni}$ leads, the conductance of the minority and majority channels were virtually the same despite the very different occupation of the two spin channels at the Fermi level. To summarize, the $I-V$ characteristics of the lead/DTE/lead system in the closed conformation appears relatively independent of both lead material and lead spin polarization, although there exist of course some differences of quantitative nature. In the open conformation, the domination of $d$-electrons in the lead material leads to sustained lower conductance also at high bias compared to the situation for $s$-dominated lead materials. The reasons for all these behaviors have been analyzed below. First, however, the effect of neglecting how finite bias affects the electronic structure of the system in the calculation the $I-V$ characteristics will be briefly addressed (right hand side of Fig. 3). 


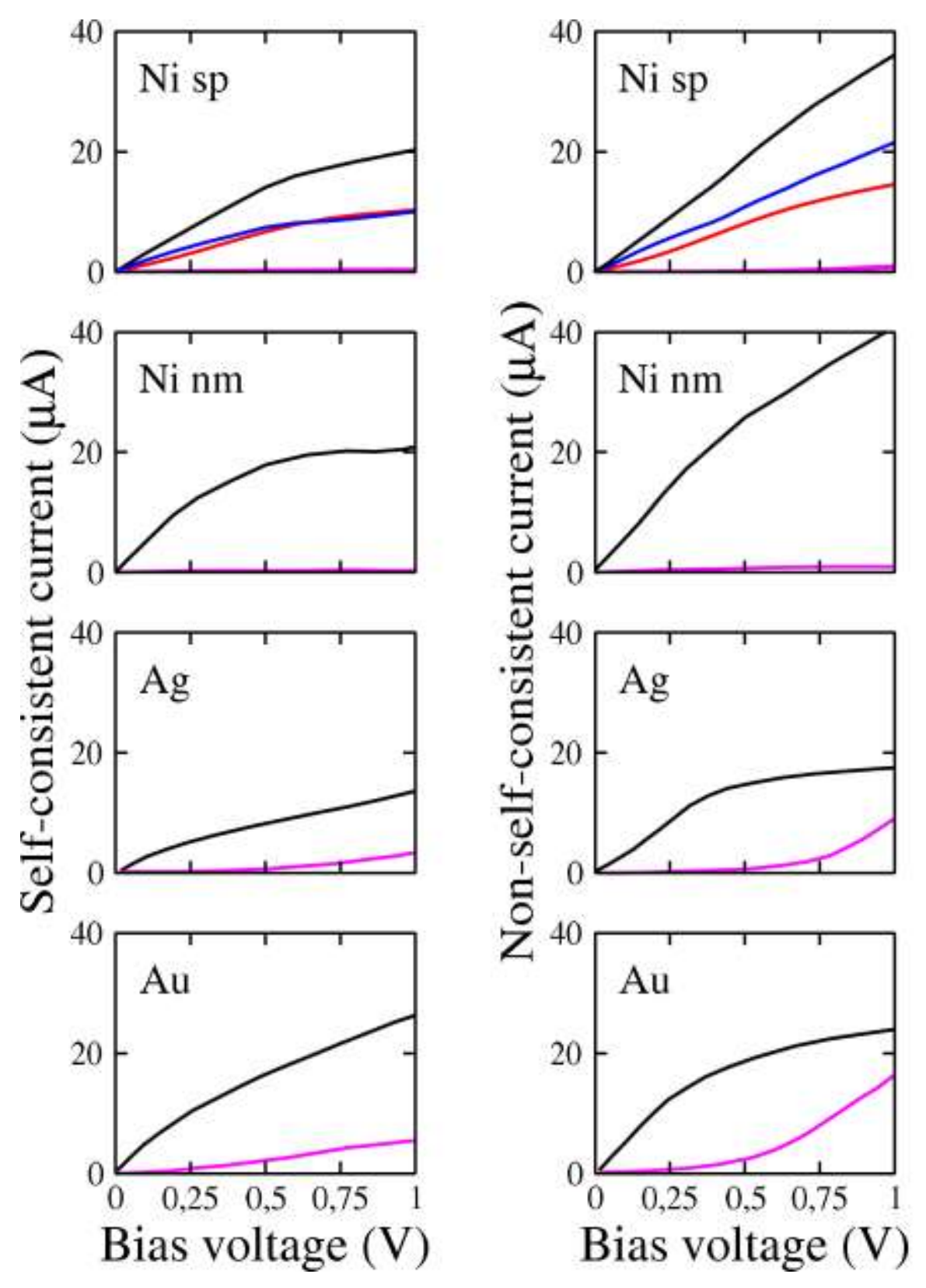

Fig. 3. (Color online) $I-V$ characteristics for the closed and the open conformations of the DTE molecule attached to four different contact materials: from top to bottom spin-polarized $\mathrm{Ni}$, nonmagnetic $\mathrm{Ni}, \mathrm{Ag}$ and $\mathrm{Au}$. The left-hand column shows the $I-V$ characteristics calculated selfconsistently and the right hand column shows the non self-consistent $I-V$ characteristics calculated by integrating the zero-bias transmission function over the relevant bias window. The black and magenta curves denote the total current for the closed and open conformation of DTE, respectively. The red and blue curves denote the majority and minority spin currents, respectively The values for the current in the open conformation of the molecule are very small in the case of Ni contacts. 
A simple and fast way to estimate the conductance at finite bias is to calculate the transmission coefficient at zero bias and then, using the Landauer-Büttiker formula [25], integrate this transmission over the relevant interval to get the finite bias conductance. However, in certain cases, if the molecular orbitals are strongly affected by the bias itself, this approach may give qualitatively wrong results. In the $\mathrm{Au}$ and $\mathrm{Ag}$ cases, the conductance of the open isomer increased quickly with bias in this approximation (Fig. 3, right column), and actually surpasses the conductance of the closed isomer at a bias of slightly more than $1 \mathrm{~V}$ [16,17], at odds with the self-consistent calculations (Fig. 3, left column). Interestingly though, for the cases of $\mathrm{Ni}$, this simplified scheme seems to work better. The conductance of the open isomer was well described, without the spurious fast increase with bias as in the $\mathrm{Au}$ and $\mathrm{Au}$ cases. However, the conductance of the closed conformation was overestimated by almost a factor of two for bias voltages above around $0.5 \mathrm{~V}$ for the cases of $\mathrm{Ni}$. Thus to this aspect, there seem to be qualitative differences in behavior between, on the one hand $\mathrm{Au}$ and $\mathrm{Ag}$, and on the other hand $\mathrm{Ni}$. In the case of the open configuration, the observed differences may lead one to conclude that the DTE molecule for some reason was less affected by the bias when attached to Ni electrodes. Such a conclusion was however not correct, as showen by analyzing the transmission spectra. 

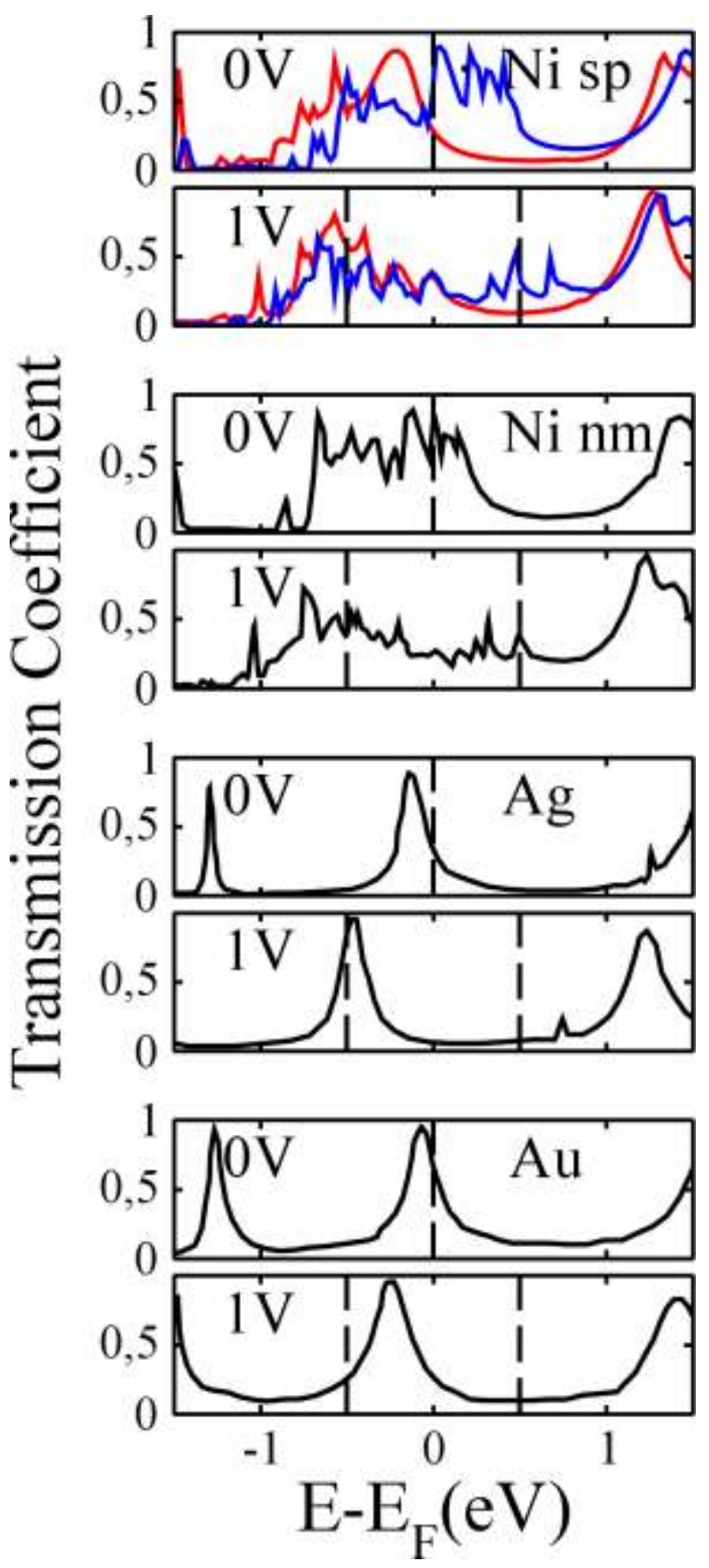

Fig. 4. (Color online) Transmission coefficients as a function of energy calculated for the closed conformation of the DTE molecule attached to four different contact materials: from top top bottom spin-polarized $\mathrm{Ni}$, nonmagnetic $\mathrm{Ni}, \mathrm{Ag}$ and $\mathrm{Au}$. For each material, the transmission functions calculated at zero bias and $1 \mathrm{~V}$ bias are shown. The red and blue curves denote the majority and minority spin transmission coefficients, respectively. The vertical dashed lines denote the integration windows used to calculate the $I-V$ characteristics.

Fig. 4 and Fig. 5 shows calculated transmission functions for all four lead materials, for the closed and open isomers respectively. The transmission is shown for zero bias as well as for a bias of $1 \mathrm{~V}$. Let us first recapitulate the main features of the transmission functions calculated with $\mathrm{Au}$ and $\mathrm{Ag}$ 
leads in order to put the results for spin-polarized Ni leads into context. It can be noted that the transmission functions for the closed conformation with $\mathrm{Au}$ and $\mathrm{Ag}$ leads were very similar. With these contact materials, a broadened molecular level was present just below the Fermi level in the closed isomer (Fig. 4). As the bias increases, this level was shifted downward in energy, but in such a way that a larger portion of the level was included in the integration window as bias increases. The net result was a significant increase of the conductance with bias. It was interesting to note that the shapes of the transmission peaks for the closed isomer does not changed with the bias, and that the molecular state showed a clear tendency to shift its energy to mainly adjust to the Fermi level of the lead with the lowest potential. The physical picture emerging is that of a conducting state extending from one lead to the other, mediated through the molecule, and that the coupling to either lead is about equal, also at finite bias (leading to a maximum transmission of almost one). In contrast, for the open isomer (Fig. 5) a broad transmission peak, approximately $1 \mathrm{eV}$ wide, resides below the Fermi level at zero bias. Only the tail of this peak reaches the Fermi level and therefore the zero-bias conductance becomes very small. As the bias increases, the broad peak narrows (this is especially evident in the case of Au leads) resulting in a consistently small conductance for the open isomer even at high bias. It is now clear why the non-self-consistent computational scheme gives qualitatively wrong results for $\mathrm{Au}$ and $\mathrm{Ag}$ - the transmission function is simply very sensitive to applied bias. Neglecting this dependence means integrating over a broad transmission peak leading to a spurious high conductance, in the case of the open isomer. Further analysis for the case of Au of the molecular electronic structure as a function of bias shows that the bias significantly polarizes the DTE molecular orbitals in the carbon backbone in the open isomer and that this is the origin of the observed changes in the transmission function [16]. 


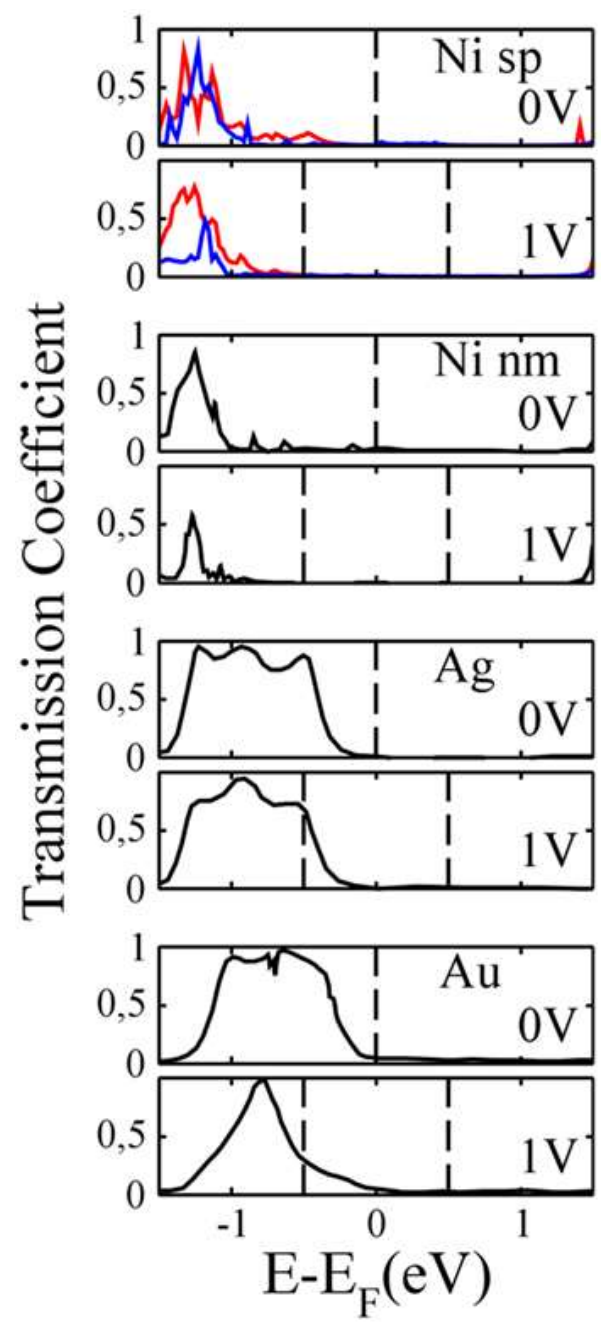

Fig. 5. (Color online) Transmission coefficients as a function of energy calculated for the open conformation of the DTE molecule attached to four different contact materials: from top top bottom spin-polarized $\mathrm{Ni}$, nonmagnetic $\mathrm{Ni}, \mathrm{Ag}$, and $\mathrm{Au}$. For each material, the transmission functions calculated at zero bias and $1 \mathrm{~V}$ bias are shown. The red and blue curves denote the majority and minority spin transmission coefficients, respectively. The vertical dashed lines denote the integration windows used to calculate the $I-V$ characteristics.

Ni was a more complex and intriguing case. For instance, the NSC scheme seems to work well for both the non-magnetic and spin-polarized cases for the open isomer, although there was no reason to believe that the DTE orbitals of the open isomer would not polarize strongly with bias also in these cases. Let us first briefly discuss the case of nonmagnetic Ni. For the closed isomer at zero bias, the transmission function had a broad jagged structure centered around the Fermi level (Fig. 4, panel 3 from the top). The jagged shapes were due to the presence of $\mathrm{Ni} d$-states in the bonding between the molecule and the leads. As the bias increases to $1 \mathrm{~V}$ (Fig. 4, panel 4 from the top), this peak was smeared out or divided into two smaller, overlapping peaks. The main reason for this 
change of the transmission function with bias was that, the states connecting the lead and molecule were relatively localized and as bias was increased, the energy of these levels will adjust to the potential shift of their respective lead $[17,26]$. The net result however was that the value of the Landauer-Büttiker integral will not change dramatically with bias, although one can see that the NSC scheme will indeed overestimate the conductance since the amplitude of the transmission function around the Fermi level is generally larger at zero bias. Thus, important changes in the electronic structure of the system were to a significant extent disguised in the calculation of the $I-V$ characteristics. For the open isomer attached to nonmagnetic Ni leads (Fig. 5, panels 3 and 4 from the top), the transmission function was very small in a wide interval around the Fermi level, leading to a very low conductance also at high bias. The comparatively localized nature of the orbitals in this system leads here to small orbital overlap across the molecule and therefore tunneling-like transmission. Furthermore, at high bias, the localized nature of the orbitals leads to highly asymmetric coupling to the left and right leads and, as a consequence, further suppressed transmission $[27,28]$.

It is now possible to discuss the spin-polarized case. It is interesting to note that the minority and majority channel transmission functions develop quite differently as bias is imposed. The closed isomer (Fig. 4, the two top panels) will be discussed first. In the region around the Fermi level, the transmission function of the majority spin channel partly resembles the transmission for the Ag and Au cases, with a clearly visible Lorentzian-like structure just below the Fermi level. The reason was that the $s$-states dominate at the Fermi level for the majority spin channel in $\mathrm{Ni}$, leading to a comparatively delocalized state coupling the leads and the molecule. As bias increases, the transmission in the majority channel gradually becomes jagged as the $d$-states increasingly start to contribute and the state localizes. In contrast, the transmission function for the minority spin channel closely resembles the transmission function for the nonmagnetic $\mathrm{Ni}$ case. As regards $I-V$ characteristics, it can thus be deduced from the transmission function that the NSC conductance of 
the minority channel at finite bias will be overestimated by about a factor of two for the closed conformation, in a manner very similar to the corresponding nonmagnetic $\mathrm{Ni}$ case. This agrees very well with what can be seen in Fig. 3, comparing the two top panels: the NSC minority spin current was calculated to be around $20 \mu \mathrm{A}$ at bias $1 \mathrm{~V}$, whereas the corresponding SC current was around 10 $\mu \mathrm{A}$. The conductance of the majority channel on the other hand was less overestimated, in a manner similar to the conductance in the Ag and Au cases. This explains the fact that the two spin channels show different conductance when calculated non-self-consistently, but have virtually the same conductance (as it happens) when calculated self-consistently.

The analysis above also enables us to explain why the conductance ratio as a function of bias decreases rapidly for the $\mathrm{Au} / \mathrm{DTE} / \mathrm{Au}$ and $\mathrm{Ag} / \mathrm{DTE} / \mathrm{Ag}$ systems but was virtually constant for the $\mathrm{Ni} / \mathrm{DTE} / \mathrm{Ni}$ systems. The main reason was the behavior of the open isomer transmission function. For $\mathrm{Au}$ and $\mathrm{Ag}$ leads, the broadened molecular levels contributing to the conductance reside mainly below the integration window, but with a significant tail reaching into the window, resulting in a small but increasing contribution with bias. For the Ni based devices, the transmission peaks were suppressed with notable transmission only outside the integration window, giving a nearly constant and very low conductance for the open isomer. Combined with the closed isomer $I-V$ characteristics, which were similar for all four discussed cases, the net result was a sustained high conductance ratio in the Ni-based devices compared to the Au and Ag-based devices.

\section{Conclusions}

In conclusion, it has been demonstrated that the two spin channels, despite the very different electronic structure and transmission functions, have very similar $I-V$ characteristics. The high similarity is accidental but leads to interesting consequences as regards predicted spin currents and magneto-resistance in this system. Over all, the DTE molecule attached to spin-polarized Ni leads exhibits $I-V$ characteristics rather similar to the Au/DTE/Au and Ag/DTE/Ag systems, and also to the hypothetical case where the Ni leads are not allowed to spin-polarize, although the transmission functions in each case are very different. 
The conductance ratio of the two isomers varies in a non-intuitive way with electrode electronic structure. A main difference between the cases with $s$-dominated lead metals (Au and Ag) compared to the $\mathrm{Ni}$ systems is that the large conductance ratio is sustained at high bias in the latter cases. In this respect, the case of spin polarized $\mathrm{Ni}$ electrodes does not directly appear as an intermediate case between $s$-dominated leads ( $\mathrm{Au}, \mathrm{Ag}$ ) and $d$-dominated leads (nonmagnetic $\mathrm{Ni}$ ). The physical origin of this property lies in the localized nature of the bonds connecting the $d$-dominated electrode material with the molecule since this leads to relative suppression of the transmission function for the open conformation. At high enough bias, both leads in the spin-polarized Ni-case exhibit typical $d$ characteristics. The sustained high conductance ratio in the system with Ni leads may be relevant for future device design. It can also be noted that with the latest measurements performed on the DTE molecule attached to $\mathrm{Au}$, the agreement between calculated and measured conductance ratios now appears entirely reasonable [10].

Further, it was found that a necessary condition for the failure of the NSC scheme is a significant polarization or shifting of the orbitals with external bias. This condition is however not sufficient. A broad transmission peak around the average Fermi level will effectively disguise the polarization and cause the NSC scheme to give nearly correct results, at least for the nonmagnetic transport properties. Obviously, the NSC scheme can be expected to work better the lower the bias is. However, as our results demonstrate, also for very low bias the NSC computational scheme will predict significant spin currents and magnetoresistance, which is qualitatively wrong judging from the self-consistent calculation. Thus, for spin-based properties, the NSC scheme gives qualitatively wrong results for the studied case.

In general, the participation of relatively localized $d$-states in the bonding between lead and molecule can be expected to generate a sustained high conductance ratio for similar molecular electronics systems, due to the way the transmission peaks are affected by localized bonds combined with bias, as demonstrated in the present work. It can therefore be speculated that similar features 
may be brought out also with other $d$-dominated lead materials such as transition metal oxides or silicides, materials which due to their chemical and structural stability are probably more suitable than metallic Ni in real applications.

\section{Acknowledgments}

This work was sponsored by the Swedish Research Links program of the Swedish Research Council, the CSIR, India, the DST, India, the Knut and Alice Wallenberg Foundation, the Swedish Royal Academy of Sciences, SeRC, and NexTec. The computations were carried out on resources provided by SNIC and HPC2N. Helpful discussions with I. Rungger and S. Sanvito are acknowledged.

\section{References}

[1] A.H. Flood, J. F. Stoddart, D.W. Steuerman, J.R. Heath, Whence molecular electronics?, Science 306 (2004) 2055-2056.

[2] I.A. Campbell, Strong and weak ferromagnetism in Ni-Fe alloys, J. Phys. F: Met. Phys. 4 (1974), L181-L183.

[3] A. Kokalj, Computer graphics and graphical user interfaces as tools in simulations of matter at the atomic scale, Comp. Mater. Sci. 28 (2003) 155-168.

[4] D. Dulic, S.J. van der Molen, T. Kudernac, H.T. Jonkman, J.J.D. de Jong, T.N. Bowden, J. van Esch, B.L. Feringa, B.J. van Wees, One-way optoelectronic switching of photochromic molecules on gold, Phys. Rev. Lett. 91 (2003) 207402.

[5] N. Katsonis, T. Kudernac, M. Walko, S.J. van der Molen, B.J. van Wees, B.L. Feringa, Reversible conductance switching of single diarylethenes on a gold surface, Adv. Mater. 18 (2006) 1397-1400. 
[6] K. Matsuda, H. Yamaguchi, T. Sakano, M. Ikeda, N. Tanifuji, M. Irie, Conductance Photoswitching of Diarylethene-Gold Nanoparticle Network Induced by Photochromic Reaction, J. Phys. Chem. C 112 (2008) 17005-17010.

[7] A.J. Kronemeijer, H.B. Akkerman, T. Kudernac, B.J. van Wees, B.L. Feringa, P.W. Blom, B. de Boer, Reversible conductance switching in molecular devices, Adv. Mater. 20 (2008) 14671473.

[8] S.J. van der Molen, J. Liao, T. Kudernac, J.S. Agustsson, L. Bernard, M. Calame, B.J. van Wees, B.L. Feringa, C. Schöneberger, Light-Controlled Conductance Switching of Ordered MetalMolecule-Metal Devices, Nano Lett. 9 (2009) 76-80.

[9] J. He, F. Chen, P.A. Liddell, J. Andreasson, S.D. Straight, D. Gust, T.A. Moore, A.L. Moore, J. Li, O.F. Sankey, S.M. Linday, Switching of a photochromic molecule on gold electrodes: single-molecule measurements, Nanotechnology 16 (2005) 695-702.

[10] E.S. Tam, J.J. Parks, W.W. Shum, Y.W. Zhong, M.B. Santiago-Berríos, X. Zheng, W. Yang, G.K.L. Chan, H.D. Abruña, D. Ralph, Single-Molecule Conductance of Pyridine-Terminated Dithienylethene Switch Molecules, ACS Nano 5 (2011) 5115-5123.

[11] J. Li, G. Speyer, O.F. Sankey, Conduction switching of photochromic molecules, Phys. Rev. Lett. 93 (2004) 248302.

[12] M. Zhuang, M. Ernzerhof, Mechanism of a molecular electronic photoswitch, Phys. Rev. B $72(2005) 073104$.

[13] M. Kondo, T. Tada, K.A. Yoshizawa, A theoretical measurement of the quantum transport through an optical molecular switch, Chem. Phys. Lett. 412 (2005) 55-59.

[14] Y. Tsuji, A. Staykov, K. Yoshizawa, Orbital view concept applied on photoswitching systems, Thin Solid Films 518 (2009) 444-447. 
[15] M. Volmer, M. Stratmann, H. Viefhaus, Electrochemical and electron spectroscopic investigations of iron surfaces modified with thiols, Surface and Interface Analysis 16 (1990) 278282.

[16] A. Odell, A. Delin, B. Johansson, I. Rungger, S. Sanvito, Investigation of the Conducting Properties of a Photoswitching Dithienylethene Molecule, ACS Nano 4 (2010) 2635-2642.

[17] A. Odell, A. Delin, B. Johansson, K. Ulman, S. Narasimhan, I. Rungger, S. Sanvito, Comparison between s- and d-electron mediated transport in a photoswitching dithienylethene molecule using ab initio transport methods, Phys. Rev. B 84 (2011) 165402.

[18] J.M. Soler, E. Artacho, J.D. Gale, A. Garcia, J. Junquera, P. Ordejon, D. Sanchez-Portal, The SIESTA method for ab initio order-N materials simulation, J. Phys.: Cond. Matt. 14 (2002) 2745-2779.

[19] A.R. Rocha, V. Garcia-Suarez, S.W. Bailey, C.J. Lambert, J. Ferrer, S. Sanvito, Towards molecular spintronics , Nature Materials 4 (2005) 335-339.

[20] A.R. Rocha, V. Garcia-Suarez, S.W. Bailey, C.J. Lambert, J. Ferrer, S. Sanvito, Spin and molecular electronics in atomically generated orbital landscapes, Phys. Rev. B. 73 (2006) 085414.

[21] I. Rungger I, S. Sanvito, Algorithm for the construction of self-energies for electronic transport calculations based on singularity elimination and singular value decomposition, Phys. Rev. B. 78 (2008) 035407.

[22] C. Toher, A. Filippetti, S. Sanvito, K. Burke, Self-interaction errors in density-functional calculations of electronic transport, Phys. Rev. Lett. 95 (2005) 146402.

[23] N. Troullier, J.L. Martins, Efficient pseudopotentials for plane-wave calculations, Phys. Rev. B 43 (1991) 1993-2006.

[24] J.P. Perdew, K. Burke, and M. Ernzerhof, Generalized gradient approximation made simple, Phys. Rev. Lett. 77 (1996) 3865-3868. 
[25] M. Büttiker, Y. Imry, R. Landauer, S. Pinhas, Generalized many-channel conductance formula with application to small rings, Phys. Rev. B 31 (1995) 6207-6215.

[26] I. Rungger, O. Mryasov, S. Sanvito, Resonant electronic states and I-V curves of Fe/MgO/Fe(100) tunnel junctions, Phys. Rev. B 79 (2009) 094414.

[27] S. Datta, Electrical resistance: an atomistic view, Nanotechnology 15 (2004) S433-S451.

[28] C.D. Pemmaraju, I. Rungger, S. Sanvito, Ab initio calculation of the bias-dependent transport properties of Mn-12 molecules, Phys. Rev. B 80 (2009) 104422. 